\title{
Inclined, Expanded and Curved Interference Pattern of Double Slit Experiment-Mathematical Description (1)
}

Hui Peng ( $\sim$ davidpeng1749@gmail.com )

https://orcid.org/0000-0002-1844-3163

\section{Research Article}

Keywords: double slit experiment, interference pattern, quantum mechanics, optics

Posted Date: July 30th, 2021

DOl: https://doi.org/10.21203/rs.3.rs-755594/v1

License: (c) (1) This work is licensed under a Creative Commons Attribution 4.0 International License.

Read Full License 


\title{
Inclined, Expanded and Curved Interference Pattern of Double Slit Experiment ---Mathematical Description (1)
}

\author{
Hui Peng \\ Davidpeng1749@gmail.com
}

\begin{abstract}
:
Young's double slit experiments express the mystery of quantum mechanics. To explore the mystery, varieties of the double slit experiments were performed. It has been shown that the following three phenomena emerged simultaneously in one double slit experiment: (1) the interference patterns incline towards the axis that perpendicular to the axis the diaphragm rotating around; (2) the interference patterns curved; (3) the distances between the fringes of the interference patterns expanded. To determine the direction the interference pattern curved towards, we proposed the right-hand rule for the clockwise rotating diaphragm and the left-hand rule for the counterclockwise rotating diaphragms. In this article we derived the mathematical formulars for the first phenomenon that interference patterns incline. We show that the inclination of the interference pattern is determined by two angles, one is the original orientation of the double slit, other one is the rotating angle of the diaphragm, either clockwise or counterclockwise.
\end{abstract}

Declaration: this work has no potential interesting conflict

Keywords: double slit experiment, interference pattern, quantum mechanics, optics

\section{Introduction}

Young's double slit experiment was first performed in 1801 [1,2], which, 100 years later, led to wave-particle duality. Feynman called the double slit experiment "a phenomenon which is impossible $[\ldots]$ to explain in any classical way, and which has in it the heart of quantum mechanics. In reality, it contains the only mystery [of quantum mechanics]." [3]. Moreover, the nature of photons truly puzzled Einstein. He wrote to M. Besso: “All these 50 years of conscious brooding have brought me no nearer to the answer to the question: What are light quanta?" [4].

In the standard double slit experiments, the characteristics of the interference patterns are described by three factors, wave length, the spacing between two slits and distance between the double slit and screen, as described by equation, $y_{\text {const }}=m \frac{\lambda}{d} L$. Note that (1) there is only one factor related to the parameter of the diaphragm of the double slit, i.e., the spacing d; (2) the fringes of the 
interference pattern distribute along a straight line; (3) the equation was derived for a special situation that the light beam is perpendicular to the plane of the diaphragm of the double slit.

We have raised a question whether the orientations of the diaphragm affect the characteristics of the interference pattern? To answer this question, several experiments have been performed [5] [6] [7] [8] [9]. We have shown experimentally that, by rotating the diaphragm of the double slit around one axis, three phenomena have been observed in the same interference pattern simultaneously: (1) the interference patterns incline towards the axis that perpendicular to the axis the diaphragm rotating around; (2) the interference patterns curved; (3) the distances between the fringes of the interference patterns expanded. To determine the direction of the interference patterns curved towards, we propose Left-hand Rule and Right-hand Rule [9].

In this article we propose mathematical formula to describe the inclination of the interference patterns of the double slit experiments.

\section{Inclination of Interference Patterns}

First let us review several examples of the inclinations. Figure 1 shows the diaphragm of the double slit.

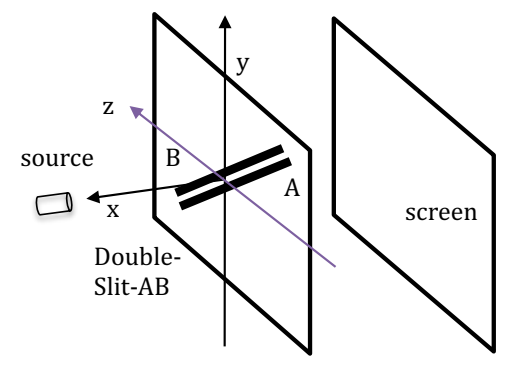

Figure 1 Schematics of double slit experiments

First, rotating the diaphragm $60^{\circ}$ and $75^{\circ}$ respectively around Y-axis counterclockwise (Figure 2).

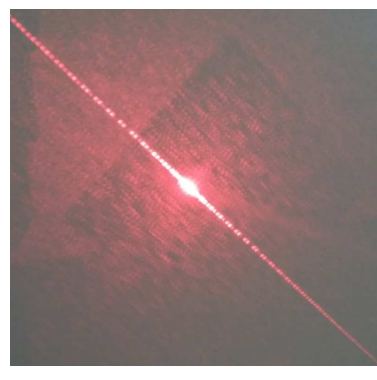

(a)

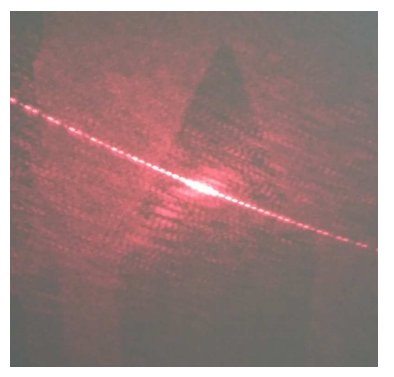

(b)

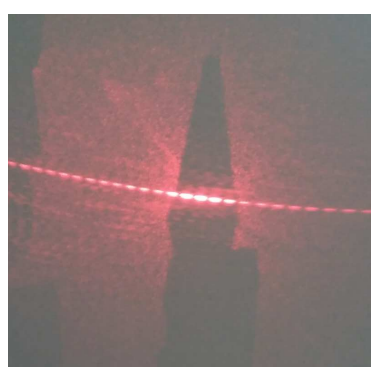

(c)

Figure 2 Rotating diaphragm around Y-axis counterclockwise: (b) $60^{\circ}$; (c) $75^{\circ}$

Figure $2 \mathrm{a}$ shows the interference pattern before the diaphragm rotating. Figure $2 \mathrm{~b}$ and $2 \mathrm{c}$ show the patterns' inclinations with difference rotation angles and support the Left-hand rule [9]

Second, rotating the diaphragm $60^{\circ}$ and $75^{\circ}$ respectively around $\mathrm{Y}$-axis clockwise. 


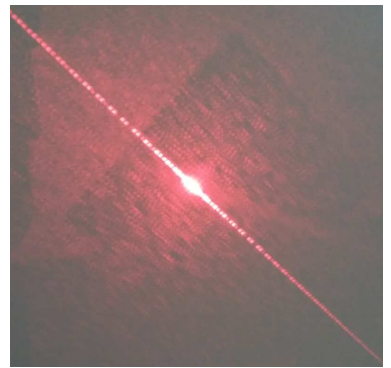

(a)

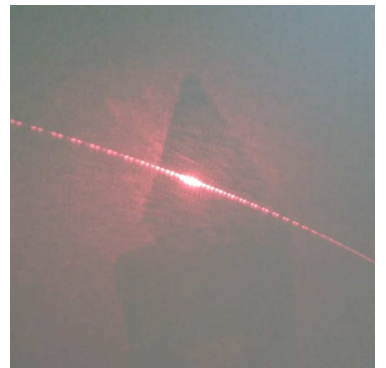

(b)

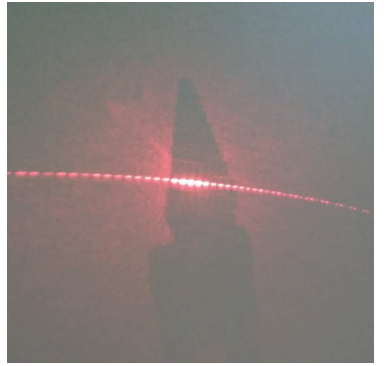

(c)

Figure 3 Rotating diaphragm around $\mathrm{Y}$-axis clockwise: (b) $60^{\circ}$; (c) $75^{\circ}$

Figure $3 \mathrm{a}$ shows the interference pattern before the diaphragm rotating. Figure $3 \mathrm{~b}$ and $3 \mathrm{c}$ show the patterns' inclinations with difference rotation angles and support the Right-hand rule [9]. After we derive the mathematical formulas for the inclination in Section 3, we will show that the experiments in Section 2 support formulas in Section 4.

\section{Derivation of Mathematical Formula for Inclination of Interference Patterns}

The phenomena take place when the diaphragms rotate, we expect that the formula relates with the rotation angle. Now we study the effects for two configurations.

\section{Configuration-1:}

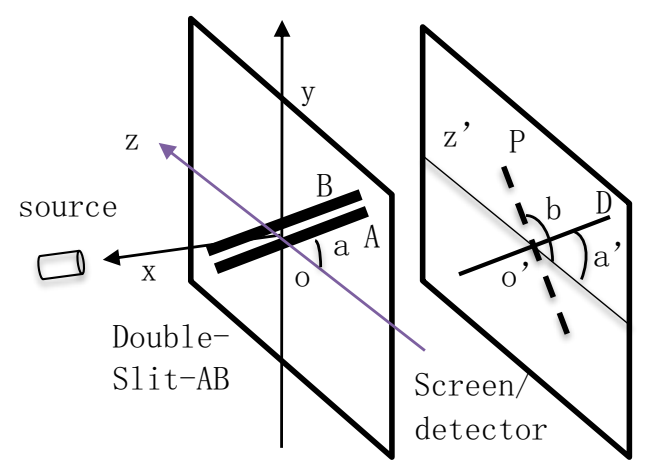

Figure 4 Schematic of Inclination of Double Slit Experiment-1

The double slit- $\mathrm{AB}$ is in the $\mathrm{Y}-\mathrm{Z}$ plane of the diaphragm. The source is on $\mathrm{X}$-axis. The angle $\angle \mathrm{a}$ is the original angle between the double alit- $\mathrm{AB}$ and $\mathrm{Z}$-axis. $\mathrm{Z}$ '-axis is the projection of $\mathrm{Z}$-axis on the detector. The "P" represents the interference pattern formed on the detector. The " $\mathrm{D}$ " represents the projection of the double slit- $\mathrm{AB}$ on the detector. The interference pattern " $\mathrm{P}$ " is perpendicular to the projection " $\mathrm{D}$ ". The angle $\angle \mathrm{b}$ is the angle between "P" and $\mathrm{Z}$ '-axis. The angle $\angle \mathrm{a}$ ' is the angle between " $\mathrm{D}$ " and Z'-axis. At the original orientation of the diaphragm, $\angle a$ ' is equal to the $\angle a$. We have

$$
\angle b=90^{\circ}+\angle a^{\prime}
$$

To describe mathematically, pick an arbitrary point " $m$ " on the "D", making a line mq perpendicular to Z'-axis and meets Z'-axis at the point “q", the line o'q is the projection of the line o'm 
on $Z^{\prime}$-axis. The angle $\angle m o^{\prime} q$ is $\angle a^{\prime}$ (Figure 5).

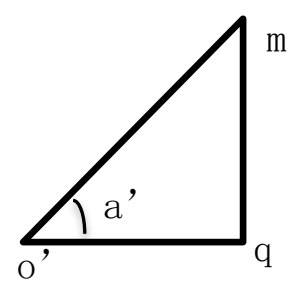

Figure 5 Schematic of projection of double slit on detector before rotating

We have,

$$
\frac{m q}{o^{\prime} q}=\tan \angle a^{\prime}
$$

When the diaphragm rotates around Y-axis counterclockwise, the point " $m$ " rotates to the point " $m$ " ".

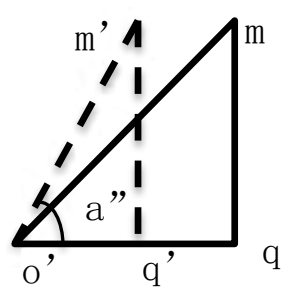

Figure 6 Schematics of projection of double slit on detector after rotating counterclockwise

The vertical length of the line $m q$ keeps the same, i.e.,

$$
m q=m^{\prime} q^{\prime}
$$

but the line $o^{\prime} q$ becomes the line $o^{\prime} q^{\prime}$ that is shorter then $o^{\prime} q$,

$$
o^{\prime} q>o^{\prime} q^{\prime}
$$

The line $o^{\prime} q$ represents the projection of the "D" on Z'-axis. The angle $\angle m^{\prime} o^{\prime} q^{\prime}$ is the angle between the projection of the "D" and Z'-axis. The $\angle m o^{\prime} q$ becomes $\angle m^{\prime} o^{\prime} q$, denoted as $\angle a$ ". We have

$$
\begin{gathered}
\angle m^{\prime} o^{\prime} q^{\prime}>\angle m o^{\prime} q \\
\angle m o^{\prime} q=\angle a^{\prime}=\angle a \\
\angle a^{\prime \prime}>\angle a^{\prime} \\
\tan \angle m^{\prime} o^{\prime} q^{\prime}=\frac{m^{\prime} q^{\prime}}{o^{\prime} q^{\prime}}=\frac{m q}{o^{\prime} q^{\prime}}
\end{gathered}
$$

Or

$$
o^{\prime} q^{\prime}=\frac{m q}{\tan \angle m^{\prime} o^{\prime} q^{\prime}} .
$$

The interference pattern is perpendicular to the projection of the double slit. Now the angle between the interference pattern "P" and Z'-axis is

$$
90^{0}+\angle m^{\prime} o^{\prime} q^{\prime}>90^{0}+\angle a .
$$

The larger the diaphragm's rotation angle, the shorter the line $o^{\prime} q^{\prime}$ and the larger $\angle m^{\prime} o^{\prime} q^{\prime}$. Thus, the interference pattern inclines to $Z^{\prime}$-axis when the diaphragm rotates. 
Next to derive the mathematical formula.

Since $m q=m^{\prime} q^{\prime}$, to find the $\angle m^{\prime} o^{\prime} q^{\prime}$, we need to find $o^{\prime} q^{\prime}$. For this aim, let us draw the projections of the line o'm' on the X-Z-plane, denoted as the line o'q". the angle of the "D" rotated counterclockwise is $\angle q^{\prime \prime} o^{\prime} q^{\prime}$ or $\angle e$ (Figure 7).

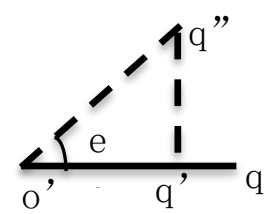

Figure 7 Schematics of projection of double slit on X-Z-plane after rotating

When the diaphragm rotates around Y-axis the angle $\angle$ e, the line o'q becomes o'q".

$$
\begin{gathered}
o^{\prime} q=o^{\prime} q^{\prime \prime} \\
\cos \angle e=\frac{o^{\prime} q^{\prime}}{o^{\prime} q^{\prime \prime}}=\frac{o^{\prime} q^{\prime}}{o^{\prime} q} \\
o^{\prime} q^{\prime}=\left(o^{\prime} q\right) \cos \angle e .
\end{gathered}
$$

Substituting Eq. (1) into Eq. (3), we obtain

$$
\frac{m q}{\tan \angle m^{\prime} o^{\prime} q^{\prime}}=\left(o^{\prime} q\right) \cos \angle e .
$$

Since $\frac{m q}{o^{\prime} q}=\tan \angle a$, substituting it into Eq. (4) we have

$$
\cos \angle e=\frac{\tan \angle a}{\tan \angle m^{\prime} o^{\prime} q^{\prime}} \text {. }
$$

Therefore

$$
\tan \angle m^{\prime} o^{\prime} q^{\prime}=\frac{\tan \angle a}{\cos \angle e} .
$$

The angle of the pattern relative to the $\mathrm{Z}$-axis is

$$
90^{\circ}+\angle m^{\prime} o^{\prime} q^{\prime}=90^{\circ}+\operatorname{act}\left[\tan \left(\frac{\tan \angle a}{\cos \angle e}\right)\right] .
$$

The larger the rotation angle $\angle e$, the larger the angle $\angle m^{\prime} o^{\prime} q^{\prime}$, namely the interference pattern inclining to Z'-axis. The angle between the "P" and Z'-axis is depending on the original angle $\angle a$ of the double slit- $\mathrm{AB}$ and the rotating angle $\angle e$ of the diaphragm.

For four extreme situations:

1) When $\angle e=0^{0}$, i.e., the diaphragm does not rotate, $\cos \angle e=1$, act $\left[\tan \left(\frac{\tan \angle a}{1}\right)\right]=\angle a$, $\angle m^{\prime} o^{\prime} q^{\prime}=\angle a$. The interference pattern stays.

2) When $\angle e=90^{\circ}$, i.e., the diaphragm rotating $90^{\circ}, \cos \angle e=0$, act $\left[\tan \left(\frac{\tan \angle a}{0}\right)\right]=90^{\circ}$, $\angle m^{\prime} o^{\prime} q^{\prime}=90^{\circ}$. The interference pattern became parallel to Z-axis. Actually, this situation will not happen, since there will be no light passing through the double slit.

3) When $\angle a=0^{0}$, i.e., the double slit is originally perpendicular to Y-axis, act $\left[\tan \left(\frac{\tan 0^{0}}{\cos \angle e}\right)\right]=0^{0}$, the interference pattern stays vertical. 
4) When $\angle a=90^{\circ}$, i.e., the double slit is originally in Y-direction, act $\left[\tan \left(\frac{\tan 90^{\circ}}{\cos \angle e}\right)\right]=90^{\circ}$, the interference pattern stays horizontal.

Next let us study the situation of rotating the diaphragm clockwise, the projection of the double slit, i.e., the line o'q" is the line o'q' as shown in Figure 8. So, we obtain the same Eq. 6.

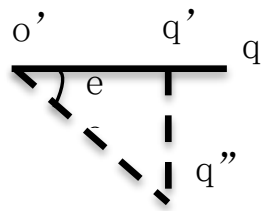

Figure 8 Schematics of the diaphragm rotating clockwise

Conclusion: The directions of the diaphragms rotate, either the clockwise or counterclockwise, have no effect on the inclination of the interference pattern.

\section{Configuration-2:}

Configurataion-2 is shown in Figure 9 below:

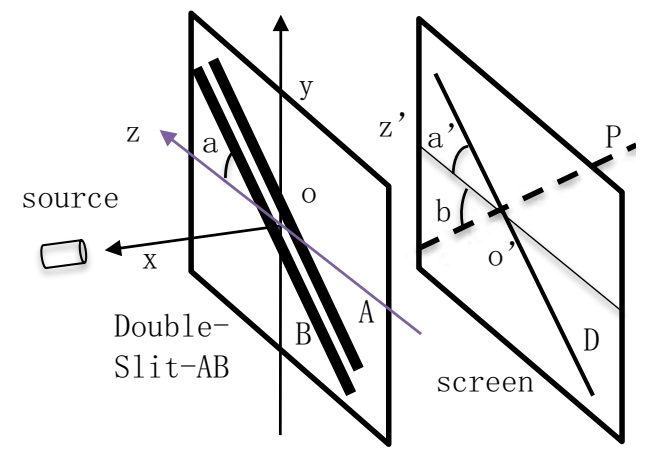

Figure 9 Schematic of Inclination of Double Slit Experiment-2

The double slit-AB is in Y-Z plane of the diaphragm. The source is on $\mathrm{X}$-axis. The angle $\angle$ a is the original angle between the double alit-AB and Z-axis. Z'-axis is the projection of Z-axis on the screen. The "P" represents the interference pattern formed on the screen. The " $\mathrm{D}$ " represents the projection of the double slit-AB on the screen. The "P" is perpendicular to the " $\mathrm{D}$ ". The angle $\angle \mathrm{b}$ is the angle between " $\mathrm{P}$ " and $\mathrm{Z}$ '-axis. The angle $\angle \mathrm{a}$ ' is the angle between " $\mathrm{D}$ " and $\mathrm{Z}$ '-axis. At the original orientation of the diaphragm, $\angle a^{\prime}$ is equal to the $\angle$ a. We have

$$
\angle b=90^{\circ}-\angle a^{\prime}
$$

To describe mathematically, pick an arbitrary point " $\mathrm{m}$ " on the $\mathrm{D}$, making a line mq perpendicular to Z'-axis and meets z'-axis at the point q. The line o'q is the projection of the line o'm on Z'-axis. The angle $\angle m o^{\prime} q$ is $\angle a^{\prime}$ (Figure 10). 


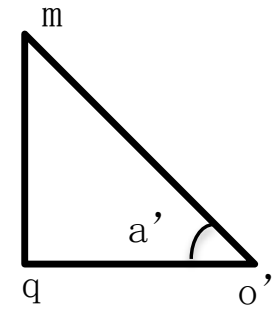

Figure 10 Schematics of projection of double slit on screen before rotating

We have,

$$
\frac{m q}{o^{\prime} q}=\tan \angle a^{\prime}
$$

When the diaphragm rotates around Y-axis clockwise, the point " $m$ " rotates to the point m' (Figure 11).

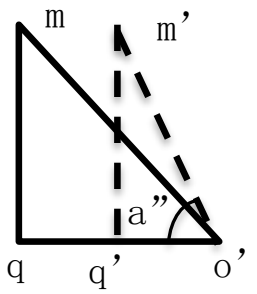

Figure 11 Schematics of projection of double slit on screen after rotating

The vertical length of the line mq keeps the same, i.e.,

$$
m q=m^{\prime} q^{\prime}
$$

but the line $o^{\prime} q$ becomes $o^{\prime} q^{\prime}$ that is shorter then $o^{\prime} q$,

$$
o^{\prime} q>o^{\prime} q^{\prime}
$$

The angle $\angle m^{\prime} o^{\prime} q^{\prime}$ is the angle between the "D", i.e., $m^{\prime} o^{\prime}$, and $Z^{\prime}$-axis. The $\angle m o^{\prime} q$ becomes $\angle m^{\prime} o^{\prime} q$, denoted as $\angle a^{\prime \prime}$. We have

$$
\angle m^{\prime} o^{\prime} q^{\prime}>\angle m o^{\prime} q
$$

and

$$
\begin{gathered}
\angle m o^{\prime} q=\angle a^{\prime}=\angle a \\
\angle a^{\prime \prime}>\angle a^{\prime} \\
\tan \angle m^{\prime} o^{\prime} q^{\prime}=\frac{m^{\prime} q^{\prime}}{o^{\prime} q^{\prime}}=\frac{m q}{o^{\prime} q^{\prime}}
\end{gathered}
$$

Or

$$
o^{\prime} q^{\prime}=\frac{m q}{\operatorname{tan\angle m^{\prime }o^{\prime }q^{\prime }} .}
$$

The interference pattern is perpendicular to the double slit. Now the angle between the "P" and Z'-axis is

$$
90^{\circ}-\angle m^{\prime} o^{\prime} q^{\prime}<90^{\circ}-\angle a .
$$


When the rotating angle of the diaphragm becomes larger, the $\angle m^{\prime} o^{\prime} q^{\prime}$ becomes larger and approaches to $90^{\circ}$. Therefore,

$$
90^{0}-\angle m^{\prime} o^{\prime} q^{\prime} \rightarrow 0^{0}
$$

Namely, the interference pattern inclines to the Z'-axis.

To find the mathematical description, we need to find the relation between $\angle m^{\prime} o^{\prime} q^{\prime}, \angle a$ and the diaphragm's rotation angle. Since $m q=m^{\prime} q^{\prime}$, to find the $\angle m^{\prime} o^{\prime} q^{\prime}$, we need to find $o^{\prime} q^{\prime}$. For this aim, we draw Figure 12 that is in the $\mathrm{X}-\mathrm{Z}$ plane.

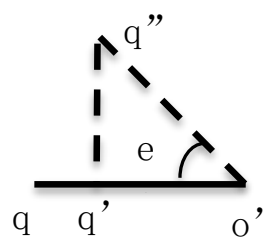

Figure 12 Schematics of projection of double slit on X-Z'-plane after rotating $\angle e$.

The $o^{\prime} q^{\prime \prime}$ is the projection of the $o^{\prime} m^{\prime}$ on X-Z'-plane. The $o^{\prime} q^{\prime}$ is the projection of $o^{\prime} q^{\prime \prime}$ on $\mathrm{Z}$ '-axis. The angle of the " $\mathrm{D}$ " rotated clockwise is $\angle q^{\prime \prime} o^{\prime} q^{\prime}$ or $\angle e$.

When the diaphragm rotates around Y-axis an angle $\angle e$ clockwise, the line $o^{\prime} q$ becomes $o^{\prime} q^{\prime \prime}$, i.e.,

$$
\begin{gathered}
o^{\prime} q=o^{\prime} q^{\prime \prime} \\
\cos \angle e=\frac{o^{\prime} q^{\prime}}{o^{\prime} q^{\prime \prime}}=\frac{o^{\prime} q^{\prime}}{o^{\prime} q} \\
o^{\prime} q^{\prime}=\left(o^{\prime} q\right) \cos \angle e .
\end{gathered}
$$

Substituting Eq. (7) into Eq. (9), we have

$$
\left(o^{\prime} q\right) \cos \angle e=\frac{m q}{\tan \angle m^{\prime} o^{\prime} q^{\prime}} .
$$

Since $\frac{m q}{o^{\prime} q}=\tan \angle a$, Eq. (10) gives

$$
\cos \angle e=\frac{\tan \angle a}{\tan \angle m^{\prime} o^{\prime} q^{\prime}}
$$

Therefore

$$
\tan \angle m^{\prime} o^{\prime} q^{\prime}=\frac{\tan \angle a}{\cos \angle e} .
$$

The angle of the interference pattern relative to the $\mathrm{Z}$-axis is

$$
90^{\circ}-\angle m^{\prime} o^{\prime} q^{\prime}=90^{\circ}-\operatorname{act}\left[\tan \left(\frac{\tan \angle a}{\cos \angle e}\right)\right] .
$$

The larger the rotation angle $\angle e$, the larger the angle $\angle m^{\prime} o^{\prime} q^{\prime}$, namely the interference pattern inclining to Z'-axis more. The angle between the interference pattern and Z'-axis is depending on the original angle $\angle a$ of the double slit-AB and the rotating angle $\angle e$ of the diaphragm.

For four extreme situations: 
1) When $\angle e=0^{0}$, i.e., the diaphragm does not rotate, $\cos \angle e=1$, act $\left[\tan \left(\frac{\tan \angle a}{1}\right)\right]=\angle a$, $\angle m^{\prime} o^{\prime} q^{\prime}=\angle a$. The interference pattern is the original one.

2) When $\angle e=90^{\circ}$, i.e., the diaphragm rotating $90^{\circ}, \cos \angle e=0$, act $\left[\tan \left(\frac{\tan \angle a}{0}\right)\right]=90^{\circ}$, $\angle m^{\prime} o^{\prime} q^{\prime}=90^{\circ}$. The interference pattern became parallel to Z-axis. Actually, this situation will not happen, since there will be no light passing through the double slit.

3) When $\angle a=0^{0}$, i.e., the double slit is originally perpendicular to Y-axis, act $\left[\tan \left(\frac{\tan 0^{0}}{\cos \angle e}\right)\right]=0^{0}$, the interference pattern stays vertical.

4) When $\angle a=90^{\circ}$, i.e., the double slit is originally in Y-direction, act $\left[\tan \left(\frac{\tan 90^{\circ}}{\cos \angle e}\right)\right]=90^{\circ}$, the interference pattern stays horizontal.

Next let us rotate the diaphragm counterclockwise, the "D", i.e., o'q", is shown in Figure 13. the projection of the o'q" is the o'q' that is the same as that in Figure 12. Therefore, we will obtain the same mathematical expression, Eq. 12.

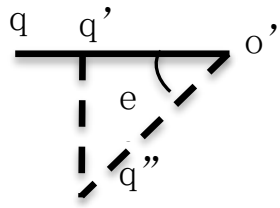

Figure 13 Schematics of the diaphragm rotating counterclockwise

Conclusion: The directions of the diaphragms rotate, either the clockwise or counterclockwise, have no effect on the inclination of the interference pattern.

\section{Testing the Mathematical Expressions}

We test the mathematical formulars with existing experiments/observations [9], in which the original angle between the double slit and $\mathrm{Y}$-axis is $\angle a=45^{\circ}$. Then rotating the diaphragms $60^{\circ}$ and $75^{0}$ respectively. There are two configurations.

Configuration-1: shown by Figure 4.

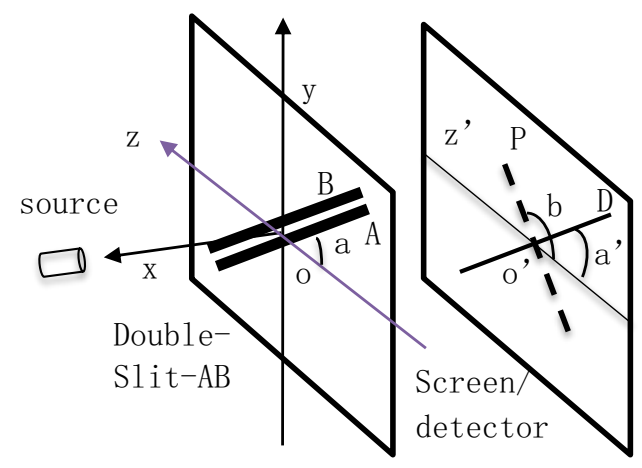

Figure 4 
We define how to measure the angle of the curved interference patterns: 1) drawing a line connecting the same-order fringes, or making the zeroth-order fringe at the center position of the line, for example, the green inclined lines in the following Figures. Then draw a horizontal line representing Z'-axis. The angle between those two lines is the inclination angle of the interference pattern relative to Z'-axis.

We have done the experiments and observed the following [9].

Rotating the diaphragm around Y-axis counterclockwise: (b) $60^{\circ}$; (c) $75^{\circ}$ (Figure 14). The angles between the interference patterns and Z-axis are shown by green lines.

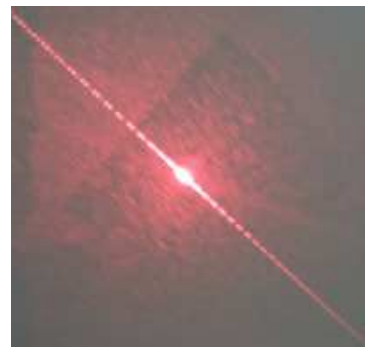

(a)

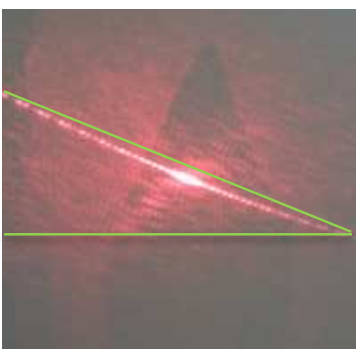

(b)

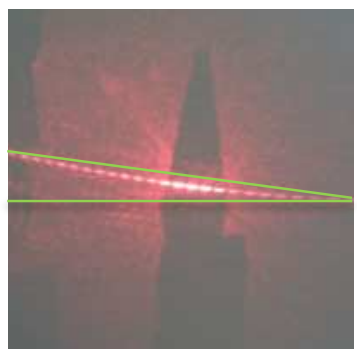

(c)

Figure 14 Rotating diaphragm around Y-axis counterclockwise: (b) $60^{\circ}$; (c) $75^{0}$

Observation: the large the rotation angle of the diaphragm, the interference patterns inclined closer to the horizontal axis. The interference pattern curved upwards, which agree with Left-hand Rule.

Rotating the diaphragm around Y-axis clockwise: (b) $60^{\circ}$; (c) $75^{\circ}$ (Figure 15). The angles between the interference patterns and Z-axis are shown by the green lines.

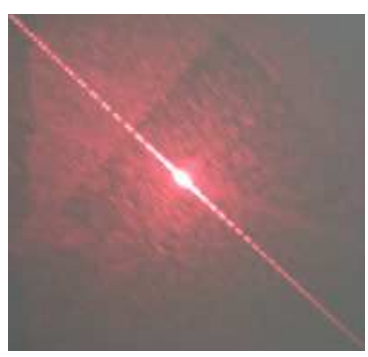

(a)

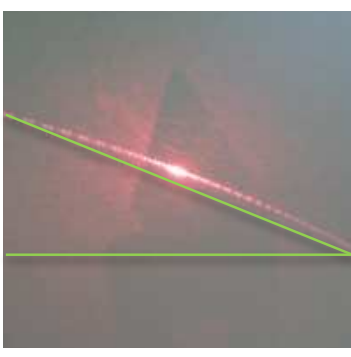

(b)

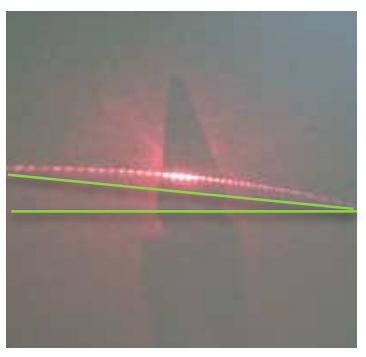

(c)

Figure 15 Rotating diaphragm around Y-axis clockwise: (b) $60^{\circ}$; (c) $75^{\circ}$

Observation: the large the rotation angle of the diaphragm, the interference patterns inclined closer to the horizontal axis. The interference pattern curved downwards, which agree with Right-hand Rule.

Eq. (6) predicts that the inclined angles of the interference patterns, attribute to the diaphragm rotating $60^{\circ}$ and $75^{\circ}$, are $153^{\circ}$ and $165^{\circ}$, respectively, either clockwise or counterclockwise,

$$
\begin{aligned}
& \angle b=90^{\circ}+\angle a^{\prime}=153^{\circ}\left(\text { For rotating } 60^{\circ}\right) \\
& \angle b=90^{\circ}+\angle a^{\prime}=165^{\circ}\left(\text { For rotating } 75^{\circ}\right)
\end{aligned}
$$

Configuration-2: shown by Figure 9 . 


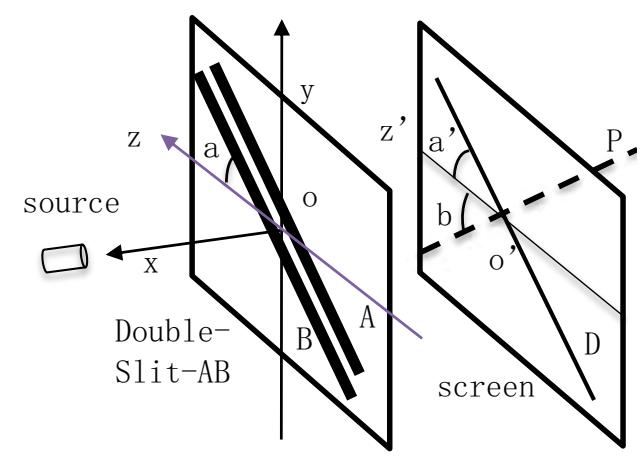

Figure 9

We have done the experiments and observed the following [9].

Rotating the diaphragm around Y-axis counterclockwise: (b) $60^{\circ}$; (c) $75^{\circ}$ (Figure 16). The angles between the interference patterns and Z-axis are shown by the green lines.

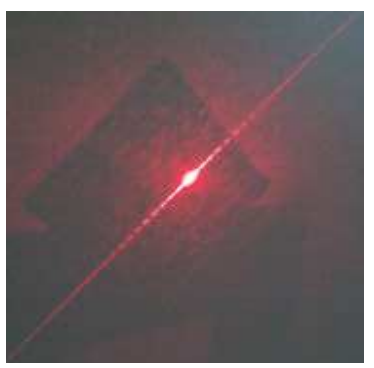

(a)

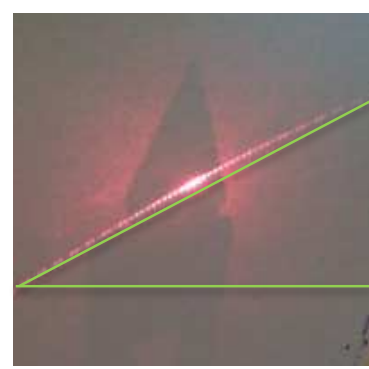

(b)

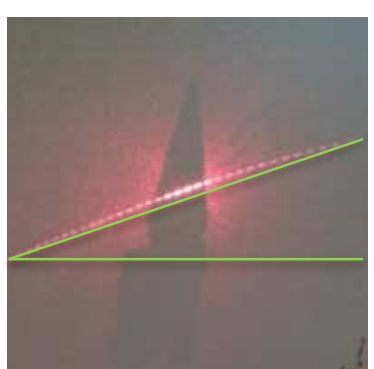

(c)

Figure 16 Rotating diaphragm around Y-axis counterclockwise: (b) $60^{\circ}$; (c) $75^{0}$

Observation: the large the rotation angle of the diaphragm, the interference patterns inclined closer to the horizontal axis. The interference pattern curved downwards, which agree with Left-hand Rule.

Rotating the diaphragm around Y-axis clockwise: (b) $60^{\circ}$; (c) $75^{\circ}$ (Figure 17). The angles between the interference patterns and Z-axis are shown by the green lines.

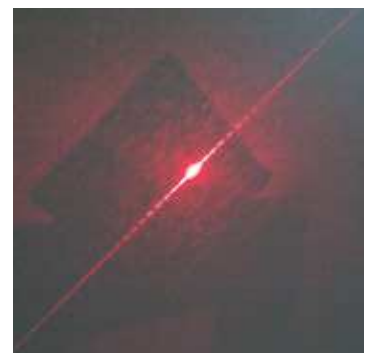

(a)

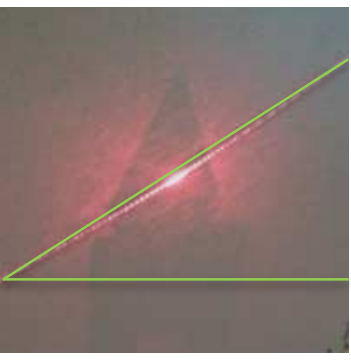

(b)

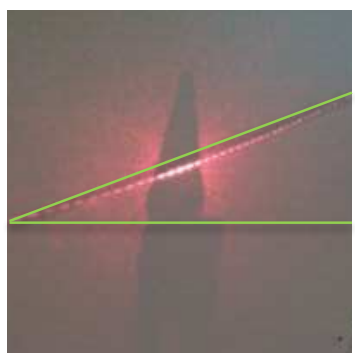

(c)

Figure 17 Rotating diaphragm around Y-axis clockwise: (b) $60^{\circ}$; (c) $75^{\circ}$

Observation: the large the rotation angle of the diaphragm, the interference patterns inclined closer to the horizontal axis. The interference pattern curved downwards, which agree with Right-hand Rule.

Eq. (12) predicts that the inclined angles of the interference patterns, attribute to the diaphragm rotating $60^{\circ}$ and $75^{\circ}$ either clockwise or counterclockwise respectively, are $27^{\circ}$ and $15^{\circ}$ respectively, 


$$
\begin{aligned}
& \angle b=90^{\circ}-\angle a^{\prime}=27^{0}\left(\text { For rotating } 60^{\circ}\right) \\
& \angle b=90^{\circ}-\angle a^{\prime}=15^{\circ}\left(\text { For rotating } 75^{\circ}\right)
\end{aligned}
$$

Conclusion: take into account the measurement accuracy, it is reasonable to suggest that the experiments/observations support the mathematical formulas derived in this article. More accurate experiments are needed.

\section{Summary}

By rotating the diaphragm of the double slit around one axis, we observe three phenomena simultaneously, namely, the interference patterns curved, expanded and inclined simultaneously [9] (see attached video). To determine the direction of the interference patterns curved towards, we proposed Left-hand Rule and Right-hand Rule. The experiments obey the rules. However, the underlying physics of the Rules is unclear.

In this article we derived the mathematical formulars for calculating the inclination angles of the interference patterns, attribute to the rotations of the diaphragms. We show that the inclination angles depend only on both the original angle of the double slit and the rotation angles of the diaphragm. The directions of the diaphragms rotating, either clockwise or counterclockwise, have no effect on the inclination.

The experimental observations support the formulars.

\section{Appendix:}

A-1: Video: the link to the video is on the last page.

Attached Video shows the evolution of the characteristics of the interference patterns of the double slit experiments.

\section{A-2: Left-Hand Rule and Right-Hand Rule to Determine Directions Patterns Curved towards}

We have shown that, attribute to the rotations of the diaphragm, either clockwise or counterclockwise, the patterns/interference patterns curved [5] [6] [7] [8].

To determine the direction the patterns curved towards, we propose Left-hand Rule and Right-hand Rule [9].

Left-hand Rule: For the curved pattern created by diaphragm rotating counterclockwise.

To determine the direction of the patterns curved towards, point the left thumb to the source, the index finger is aligned with the direction of the original pattern, and the middle finger will point in the direction of the patterns curved towards, which is attribute to the counterclockwise rotation of the diaphragm. 


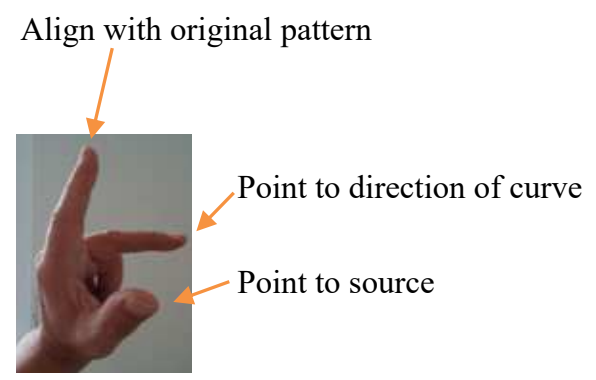

Figure A-1 Left-hand Rule

Right-hand rule: For the curved pattern created by diaphragm rotating clockwise.

To determine the direction of the patterns curved towards, point the right thumb in the direction of the source, the index finger is aligned with the direction of the original pattern, and the middle finger will point in the direction of the patterns curved towards, which is attribute to the clockwise rotation of the diaphragm.

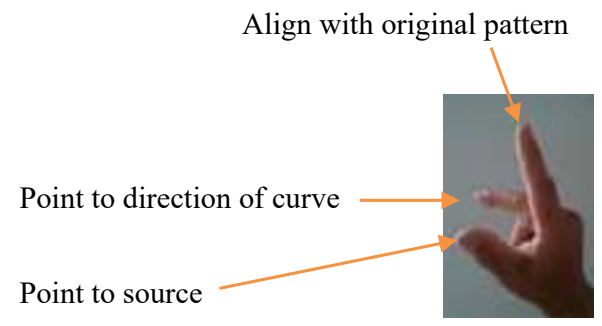

Figure A-2 Right-hand Rule

References

[1] A. Ananthaswamy, “Through Two Doors at Once”, Dutton, New York, NY, (2018).

[2] A. Robinson, “The Last Man Who Knew Everything”. New York, NY: Pi Press., (2006).

[3] R. Feynman, R. Leighton, and M. Sands, “The Feynman Lectures on Physics” (Addison-Wesley, Reading, 1966), Vol. 3.

[4] S. Rashkovskiy, Is a rational explanation of wave-particle duality possible? arXiv 1302.6159 [quant-ph] 2013.

[5] Hui Peng, "Novel Double Slit and Cross-Double Slit Experiments--- Interference Patterns Depending on Orientation of Diaphragm”, Research Square, preprint, DOI: https://doi.org/10.21203/rs.3.rs-653201/v1, 2021.

[6] Hui Peng, "Curved Interference Patterns---Novel Double Slit and Cross-double Slit Experiments”, Research Square, preprint, DOI: https://doi.org/10.21203/rs.3.rs-677223/v1. 2021.

[7] Hui Peng, "Novel Mystery of Double Slit/Cross-double Slit Experiments--- Curved Interference Pattern and Interference Pattern Depending on Orientation of Diaphragm”, Research Square, preprint, DOI: https://doi.org/10.21203/rs.3.rs-701216/v1, 2021. 
[8] Hui Peng, "Novel Universal Phenomena of Single Slit, Double Slit, Cross-double Slit and Triple Slit Experiments --- Curved Patterns and Orientation-Dependence of Patterns”, Research Square, preprint, DOI: https://doi.org/10.21203/rs.3.rs-722731/v1. 2021.

[9] Hui Peng, "Novel Double Slit Experiments: Right-hand Rule and Left-hand Rule ---Interference Patterns Curved, Expanded and Inclined Simultaneously”, Research Square, preprint, DOI: https://doi.org/10.21203/rs.3.rs-737506/v1, 2021.

Note:

Please see the next page for the link to the attached video which shows the evolution of the interference pattern of the novel double slit experiments. The video shows how the characteristics of the interference patterns vary when the diaphragm is rotating. We start from the original orientation of the diaphragm, namely, the laser beam is perpendicular to the plane of the diaphragm. The original interference pattern is shown.

Then rotating the diaphragm around the vertical direction counterclockwise. The interference patterns change gradually in three aspects continuously:

First aspect, the whole pattern is inclined towards the horizontal axis, Z-axis.

Second aspect, the distances between fringes are expanded.

Third aspect, the patterns curve upwards.

Then go back to the original orientation and rotate the diaphragm clockwise. The interference patterns still vary in three aspects: First and Second aspects are the same. Third aspect, the patterns curve, but to opposite direction, i.e., downward. 


\section{Supplementary Files}

This is a list of supplementary files associated with this preprint. Click to download.

- 00curvedexpandedandinclinedinterferencepatternofdoubleslitexperiments.mp4 